\title{
Doppler Correction to Improve TS-ESPRIT Algorithm Used in NULA
}

\author{
Youssef Fayad, Caiyun Wang, and Qunsheng Cao
}

\begin{abstract}
In this paper a modified ESPRIT algorithm based on time subspace (T-ESPRIT) and spatial subspace of estimate 1D-DOA (elevation) of a radiated source to increase the estimation accuracy with low computational load is introduced. Firstly, this algorithm treats with non uniform linear (NULA) array as a multiple sub-uniform linear arrays (ULA) with the common reference point, and the T-ESPRIT method is applied within each sub-ULA. Secondly, in order to increase the estimation accuracy, the estimated DOA is corrected with Doppler frequency (fd) which induced by target movement. Moreover, the proposed algorithm is combined the refined $\mathrm{T}$ ESPRIT method with time differential of arrival (TDOA) technique to form TS-ESPRIT algorithm which calculates an optimum DOA. The estimated results are better than the traditional ESPRIT methods leading to the estimator performance enhancement.
\end{abstract}

Index Terms-DOAE, t-esprit, Doppler frequency, TDOA.

\section{INTRODUCTION}

Great efforts have been done to improve DOAE accuracy via applying ESPRIT for uniform linear array (ULA) [1]-[7] and non-uniform linear array (NULA) [8]-[11].

In previous work, the method involved in both the temporal and spatial resolution (TS-ESPRIT) had been introduced to realize subspace approach by combining the S-ESPRIT method with the T-ESPRIT [1] , [2], [12] which realized high estimation accuracy with low computational load due to benefit from the advantages of subspaces and multi-resolution techniques.

In this paper a new idea is presented based on spatial subspace concept not on spatial sampling concept which had been used in [1], [8]-[11]. Spatial subspace is realized by divided the NULA to multiple ULAs with the same reference point and compute DOAE by applying T-ESPRIT for each separately, but in parallel with the others. Then, the effect of Doppler frequency on the T-ESPRIT method is explained in order to refine the DOAE. Finally, the TDOA technique [13], [14] is applied and combined the multiple sub-arrays to calculate the optimum DOAE value, which realizes time and space parallel processing, so that it obtains to enhance the estimation accuracy and reduce computational load.

Manuscript received June 23, 2015; revised August 25, 2015.

Youssef Fayad and Qunsheng Cao are with College of Electronic and Information Engineering, Nanjing University of Aeronautics\& Astronautics, Nanjing 210016, China (e-mail: yousseffyad@nuaa.edu.cn, qunsheng@nuaa.edu.cn).

Caiyun Wang is with College of Astronautics Nanjing University of Aeronautics and Astronautics, Nanjing 210016, China (e-mail: wangcaiyun@nuaa.edu.cn)

\section{PRoposed Algorithm}

\section{A. The Measurement MODEL}

In this model, the transmission medium and the signals are assumed to be the same as [1], and [12]. Assume there is a number antenna element, and it is divided into $\mathrm{L}$ number multiple uniform linear antenna (ULA) each with total $\mathrm{N}$ number antenna element in receiving system shown in Fig. 1. And there are $\mathrm{K}$ number emitting sources, then the output complex signal $\mathrm{z}_{n, l}(t)(n=1,2, \ldots, N, l=1, \ldots, L)$ at $(n, l)$ sensor at time $t$ can be written as,

$$
z_{n, l}(t)=\sum_{k=1}^{K} a_{n, l}\left(\theta_{k}\right) s_{k}(t)+w_{n, l}(t)
$$

where $S_{k}(t)$ is the signal of the $k^{\text {th }}$ source at time $t$. $a_{n, l}\left(\theta_{k}\right)=e^{j \frac{2 \pi \Delta_{n, l}}{\lambda} \sin \theta_{k}}$ is the sensor element response at frequency $\omega_{0}=2 \pi f$ corresponding to propagation delay between a reference point at $(n, l)$ sensor for the $k^{\text {th }}$ wave front impinging on the array from direction $\theta_{k}, \Delta_{n, l}$ is the displacement of the $n^{\text {th }}$ element in the $l^{\text {th }}$ sub-ULA with respect to the reference point (R.P). The receiving model for each $l$ sub-ULA can be written as in matrix form

$$
\left[Z_{n, l}(t)\right]=[A][S(t)]+\left[W_{n, l}(t)\right]
$$

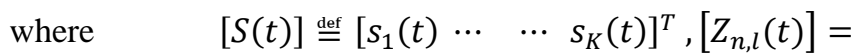

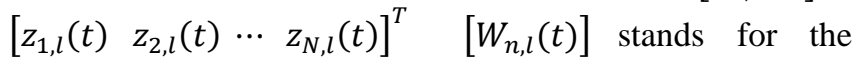
additive white Gaussian noise (AWGN), so that

$$
[A]=\left[\begin{array}{ccc}
a_{1, l}\left(\theta_{1}\right) & \cdots & a_{1, l}\left(\theta_{k}\right) \\
\vdots & \ddots & \vdots \\
a_{N, l}\left(\theta_{1}\right) & \cdots & a_{N, l}\left(\theta_{k}\right)
\end{array}\right]
$$

\section{B. Time and Spatial Subspaces Technique}

Fig. 1 shows the NULA, the displacement for each subULA element from the reference point is computed as follow

$$
\Delta_{n, l}=\left((n-1) \cdot \Delta_{1}\right)+\left((l-1) \cdot\left[(N-1) \cdot \Delta_{1}+\Delta_{2}\right]\right)
$$

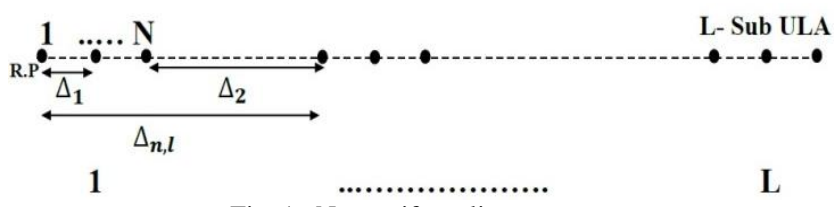

Fig. 1. Non-uniform linear array.

For the Electronic Scanning Beam (ESB) with width 
$\nabla \theta_{a z}$ which scans along angle vertically $\left(90^{\circ}\right)$ in each scan, dwell interval $\left(T_{D}\right)$ is calculated as follow [15]:

$$
T_{D}=\frac{\nabla \theta_{a z} \times 60}{90 \times \vartheta}
$$

where $\vartheta$ is the number of hits per minute,

Apply the T-ESPRIT method for each sub-ULA, each receiving signal measurement value through $m^{\text {th }}$ subspace is given as,

$$
\left[z_{n, l}^{m}(\tau)\right]=\left[A^{m}\right] s^{m}(\tau)+\left[w_{n, l}^{m}(\tau)\right]
$$

$\left[Z_{n, l}(\tau)\right] \stackrel{\text { def }}{=}$

$\left[\begin{array}{ccccccccc}\mathbf{z}_{1, l}^{1}(\mathbf{0}) & \ldots & \mathbf{z}_{1, l}^{1}\left(\boldsymbol{\tau}_{1}\right) \ldots \ldots & \mathbf{z}_{\mathbf{1}, l}^{m}(\mathbf{0}) & \ldots & \mathbf{z}_{1, l}^{m}\left(\boldsymbol{\tau}_{1}\right) \ldots \ldots \mathbf{z}_{1, l}^{M}(\mathbf{0}) & \ldots & \mathbf{z}_{1, l}^{M}\left(\boldsymbol{\tau}_{1}\right) \\ \vdots & \ddots & \vdots & \vdots & \ddots & \vdots & \vdots & \ddots & \vdots \\ \mathbf{z}_{N, l}^{1}(\mathbf{0}) & \ldots & \mathbf{z}_{N, l}^{\mathbf{1}}\left(\boldsymbol{\tau}_{1}\right) \ldots \ldots & \mathbf{z}_{N, l}^{m}(\mathbf{0}) & \ldots & \mathbf{z}_{N, l}^{m}\left(\boldsymbol{\tau}_{1}\right) \ldots \ldots & \mathbf{z}_{N, l}^{M}(\mathbf{0}) & \ldots & \mathbf{z}_{N, l}^{M}\left(\boldsymbol{\tau}_{1}\right)\end{array}\right]$

where $m=1,2, \ldots, M$ snapshots at each time $T=T_{D} / M$ second, $r$ is the number of picked up data enclosed by each snapshot $m$ with time period $\tau=\frac{T}{r}, \tau_{1}=(r-1) \tau$.

For the $m^{\text {th }}$ subspace data matrix can be expressed as,

$$
\left[z_{n, l}^{m}(\tau)\right]=\left[\begin{array}{ccc}
\mathrm{z}_{1, l}^{m}(0) & \ldots & \mathrm{z}_{1, l}^{m}\left(\tau_{1}\right) \\
\vdots & \ddots & \vdots \\
\mathrm{z}_{N, l}^{m}(0) & \ldots & \mathrm{z}_{N, l}^{m}\left(\tau_{1}\right)
\end{array}\right]
$$

The matrix $\left[z_{n, l}^{m}(\tau)\right]$ dimension is $(N \times r)$ due to apply TESPRIT algorithm.

For spatial sampling (S-ESPRIT) [1] the sensor element response is given by,

$$
a_{n}\left(\theta_{k}\right)=a_{n \Delta_{1}}\left(\theta_{k}\right) \otimes a_{n \Delta_{3}}\left(\theta_{k}\right)
$$

where $\otimes$ denotes the Kronker product, and $\Delta_{3}$ is the distance between any element in sub-array $(l)$ and its counterpart in sub-array $(l-1)$. And consequently,

$$
\left[z^{m}(\tau)\right]=\left[\begin{array}{ccc}
\mathrm{z}_{1,1}^{m}(0) & \ldots & \mathrm{z}_{1,1}^{m}\left(\tau_{1}\right) \\
\vdots & \vdots & \vdots \\
\mathrm{z}_{N, 1}^{m}(0) & \ldots & \mathrm{z}_{N, 1}^{m}\left(\tau_{1}\right) \\
\vdots & \vdots & \vdots \\
\mathrm{z}_{N, L}^{m}(0) & \cdots & \mathrm{z}_{N, L}^{m}\left(\tau_{1}\right)
\end{array}\right]
$$

So, when we apply TS-ESPRIT method; the resulted subspace data matrix dimension is $(N L \times r)$ for each $m$ subspace. Thus, it is clear from Eqs. (8) and (10) that the combination of T-ESPRIT with the spatial subspace reduces the calculations load and consequently achieving time saving with the same number array elements.

The ESPRIT algorithm is based on a covariance formulation, that is,

$$
\begin{gathered}
\hat{R}_{z z} \bar{E}=\sum_{w} \bar{E} \Lambda \\
\hat{R}_{z z} \stackrel{\text { def }}{=} E\left[Z_{n, l}^{m}(\tau) Z_{n, l}^{m}(\tau)^{H}\right]=A \hat{R}_{s S} A^{H}+\sigma^{2} \Sigma_{w} \\
\hat{R}_{s S}=E\left[S(\tau) S^{H}(\tau)\right]
\end{gathered}
$$

The superscript $H$ denotes complex conjugate transpose. Compute the Eigen decomposition,

$$
\widehat{R}_{z z} \stackrel{\text { def }}{=} \widehat{E}_{\mathrm{S}} \Lambda \widehat{\mathrm{E}}_{\mathrm{S}}^{H}+\sigma^{2} \widehat{E}_{w} \Lambda \widehat{\mathrm{E}}_{w}^{H}
$$

where the eigenvalues are ordered $\lambda_{1}>\lambda_{2}>\cdots>\lambda_{K}>$ $\lambda_{K+1}>\lambda_{K+1}=\cdots=\lambda_{N}=\sigma^{2}$. The eigenvectors $\widehat{E}_{\mathrm{S}}=\left[\hat{e}_{1}, \hat{e}_{2}, \cdots, \hat{e}_{K}\right]$ for larger $K$ eigenvalues spans the signal subspace, the rest $N-K$ smaller eigenvalues $\widehat{\mathrm{E}}_{\mathrm{w}}=$ $\left[\hat{\mathrm{e}}_{\mathrm{K}+1}, \cdots, \hat{\mathrm{e}}_{\mathrm{N}}\right]$ spans the noise subspace which is orthogonal to the signal subspace. Therefore, there exist a unique nonsingular matrix $Q$ such that,

$$
\widehat{E}_{S}=[A] Q
$$

In (15) let $A_{P 1}$ and $A_{P 2}$ be the first and the last $(N-1)$ rows of $A$ respectively, they differ by the factor $\Delta p_{k}^{l}=e^{j \frac{2 \pi \Delta}{\lambda} \sin \theta_{k}^{l}}, \quad \Delta=\Delta_{n+1, l+1}-\Delta_{n, l} \quad$ is the internal translational invariance for each $(l)$ sub-ULA but with respect to the reference point. by substituting at Eq.(4) it is found that $\Delta=\Delta_{1}$. So $A_{P 2}=A_{P 1} \emptyset_{P}$, where $\emptyset_{P}$ is the diagonal matrix with diagonal elements $\Delta p_{k}^{l}$. Consequently, $\widehat{\mathrm{E}}_{\mathrm{P} 1}$ and $\widehat{\mathrm{E}}_{\mathrm{P} 2}$ will be the first and the last $(N-1)$ sub-matrices formed from $\widehat{\mathrm{E}}_{\mathrm{S}}$. Then the diagonal elements $\Delta \mathrm{p}_{\mathrm{k}}$ of $\emptyset_{\mathrm{P}}$ are the eigenvalues of the unique matrix $\Psi_{\mathrm{P}}=\mathrm{Q}^{-1} \emptyset_{\mathrm{P}} \mathrm{Q}$, that satisfies,

$$
\widehat{E}_{\mathrm{P} 2}=\widehat{E}_{\mathrm{P} 1} \Psi_{P}
$$

Therefore,

$$
\arg \left(\Delta p_{k}^{l}\right)=\frac{2 \pi \Delta}{\lambda} \sin \theta_{k}^{l}
$$

Then,

$$
\hat{\theta}_{\mathrm{k}}^{\mathrm{l}}=\sin ^{-1}\left\{\frac{\lambda}{2 \pi \Delta} \arg \left(\Delta \mathrm{p}_{\mathrm{k}}^{\mathrm{l}}\right)\right\}
$$

\section{Doppler Correction}

The moving target echo signal is shifted by the Doppler Effect. The more accurate T-ESPRIT algorithm should consider the effect of the Doppler frequency shift due to the target movement. So,

$$
\arg \left(\Delta p_{k}^{l}\right)=\frac{2 \pi \Delta}{\grave{\lambda}} \sin \theta_{k}^{l}
$$

where $\grave{\lambda}$ is the wavelength of the received wave which differs from the transmitted wavelength because of the Doppler frequency $f_{d}$ caused by the target moving velocity $\vec{v}_{S}[16]-[18]$.

$$
\grave{\lambda}=\frac{\lambda\left(c+v_{s}\right)}{c}
$$

Substituting into (19), then,

$$
\arg \left(\Delta p_{k}^{l}\right)=\frac{2 \pi \Delta c}{\lambda\left(c+v_{s}\right)} \sin \theta_{k}^{l}
$$

and,

$$
\left|\vec{v}_{s}\right|=\frac{c \cdot f_{d}}{2 f \cdot \cos \alpha}
$$

where, $\alpha$ is the angle between the direction of propagation 
and the target velocity vector $\vec{v}_{s}$.

So

$$
\arg \left(\Delta p_{k}^{l}\right)=\frac{2 \pi \Delta \cdot(2 f \cdot \cos \alpha)}{\lambda\left(2 f \cdot \cos \alpha+f_{d}\right)} \sin \theta_{k}^{l}
$$

Then,

$$
\hat{\theta}_{k}^{l}=\sin ^{-1}\left\{\frac{\lambda}{2 \pi \Delta} \cdot\left(\frac{2 f \cdot \cos \alpha+f_{d}}{2 f \cdot \cos \alpha}\right) \cdot \arg \left(\Delta p_{k}^{l}\right)\right\}
$$

It is obvious found from Eqs. (22) and (24) that if $\vec{v}_{s}=0$, it will realize for a stationary target indicated in Eq. (18).

\section{Optimal DOAE calculation}

For ULA groups shown in Fig. 2, the $l^{\text {th }}$ TDOA for the $k^{\text {th }}$ received signal from the reference antenna is calculated as follow [14];

$$
\delta_{k}^{1 l}=\frac{\Delta_{n, l}}{c} \cos \hat{\theta}_{k}^{l}
$$

where, $\hat{\theta}_{k}^{l}$ is calculated for the $l^{t h}$ sub-array as in Eq. (24).

So, the optimal DOAE value is measured at the center of the ULA base line $\Delta_{n, l}=d_{\text {opt }}$ at which $\delta_{k}^{1 l}=\delta_{o p t}=\frac{\delta_{k}^{1 L}}{2}$. So,

$$
\hat{\theta}_{k}^{0}=\cos ^{-1} \frac{c \cdot \delta_{o p t}}{d_{o p t}}
$$

Fig. 3 represents the diagram of time and spatial subspace (TS-ESPRIT) proposed algorithm.

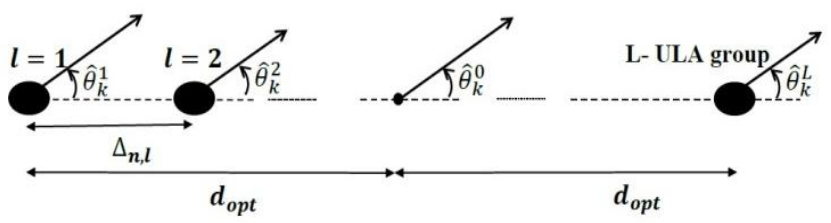

Fig. 2. Sub uniform linear array groups arrangement.

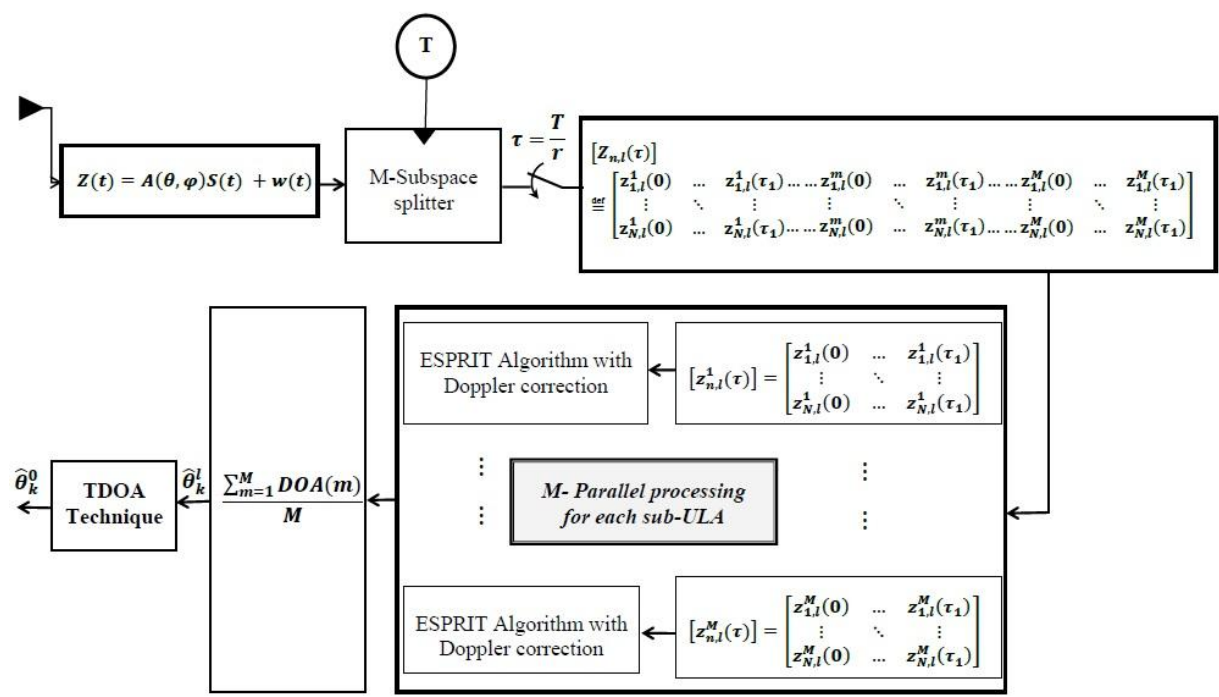

Fig. 3. The proposed TS-ESPRIT method with spatial subspace technique.

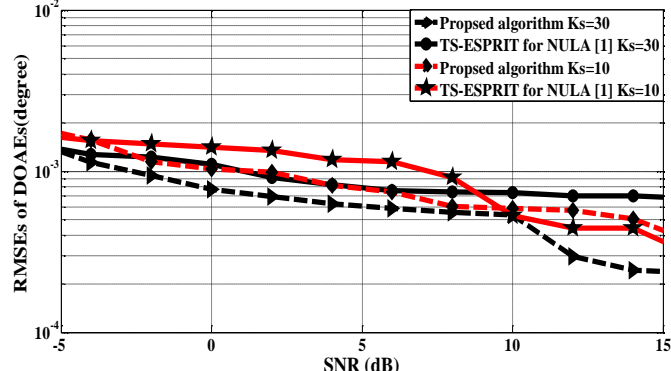

Fig. 4. RMSEs vs. SNR for the TS-ESPRIT and the proposed algorithm $\theta=34^{\circ}, k_{\mathrm{s}}=10$, and 30 .

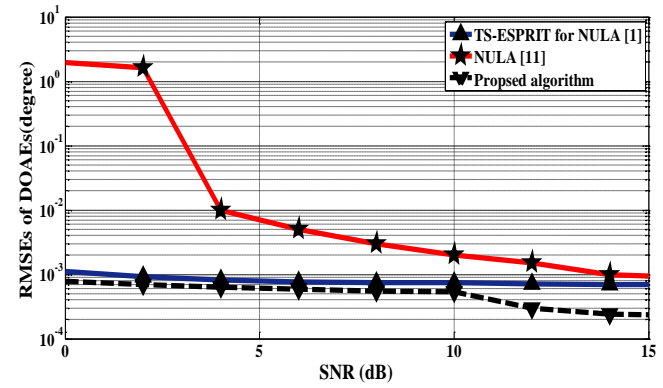

Fig. 5. RMSEs vs. SNR for the proposed method against different ESPRIT methods at $\theta=40^{\circ}, k_{\mathrm{s}}=25$.

\section{Simulation Results AND COMPARISONS}

Considering one-dimensional (1D) DOAE process with AWGN, the simulation parameters are the same as [1], $\mathrm{T}_{\mathrm{D}}=1 \mathrm{msec}, \vartheta=1000 \mathrm{scan} / \mathrm{min}$, number of hits $=4$, $v_{s}=250 \mathrm{~m} / \mathrm{sec}, f=3 \mathrm{GHz}, \alpha=0^{\circ}\left(f_{\mathrm{d}}=5000 \mathrm{~Hz}\right)$ and $f_{\mathrm{s}}=$ $25 \mathrm{MHz}$. Assuming total 25 temporal snapshots, pickup enclosed data $r=20$ times, $\nabla \theta_{a z}=1.5^{\circ}, \Delta_{1}=0.5 \lambda, \Delta_{2}=30 \times$ $\Delta_{1}$ which gave the best results in [1], 200 independent Montecarlo simulations, and SNR is chosen as -5 to $15 \mathrm{~dB}$ In order to validate the proposed algorithm, it is used in the NULA case. Then, the resulted error is compared with results of different ESPRIT methods. Fig. 4 displays the comparison between the RMSEs of TS-ESPRIT and the proposed algorithm for the NULA with 15 elements $(N=5$, $L=3), k_{s}=10$ and $k_{s}=30$ at $\theta=34^{\circ},\left(k_{s}=\frac{\Delta_{3}}{\Delta_{1}}\right)$. The accuracy improvement of the DOAE using proposed algorithm has been confirmed by comparing its resulted RMSEs with their counterparts of space multi resolution ESPRIT (S-ESPRIT) algorithm used in [11], and TSESPRIT used in [1]. Fig. 5 is comparison of DOAE for the 
NULA two methods with $N=4$ elements for $k_{s}=25$ at $\theta=40^{\circ}$ as same in [1], [11].

Table I represents the computational time and complexity of the proposed method in term of number of flip-flops. It is obvious that the computational load has been reduced as a result of employing the spatial subspaces concept for each $m$ subspace $\left[z_{n, l}^{m}\right]_{(\mathrm{N} \times r)}$ in parallel.

The results are shown in Figs. 4 and 5 show that the errors of the proposed algorithm are less than the errors of S-ESPRIT algorithm [11] and TS-ESPRIT algorithm [1] especially at low SNR. This upgrade has been occurred due to the increase of DOAE accuracy when combining the TESPRIT with spatial subspace algorithm which decreases the errors caused by the model nonlinearity effect and increases the resolution of phase deference measurement. Additionally, this improvement is due to Doppler correction which reduces the DOAE uncertainty associated with effect of the target movement.

TABLE I: COMPARISON OF THE REQUIRED COMPUTATION TIME AND COMPLEXITY

\begin{tabular}{ccc}
\hline & TS- ESPRIT & Proposed algorithm \\
computational & 1.374 & 0.523 \\
time (msec) & & \\
complexity & $O\left(M+(N L)^{2} r+(N L)^{3}\right)$ & $O\left(M+N^{2} r+N^{3}\right)$ \\
\hline \hline
\end{tabular}

It is also noticed from Fig. 4 that the estimation accuracy increases with increasing the distance between sub-ULA groups. Table I indicates that the proposed algorithm requires $O \quad\left(M+N^{2} r+N^{3}\right) \quad$ flops, while the TS- ESPRIT algorithm needs $O\left(M+(N L)^{2} r+(N L)^{3}\right)$ flops [19]. Also it notes from this Table that due to parallel processing the developed ESPRIT algorithm requires only about $38 \%$ of the computational time that required in the TS- ESPRIT algorithm. Additionally, due to use the same reference point, this algorithm enables reducing the effect of losing any sub-array output for any reason. Simply, we have found the developed ESPRIT method achieved success into increasing the DOAE accuracy with low computational load leading to increase the estimator efficacy.

\section{CONCLUSIONS}

In this paper, a new ESPRIT method is developed based on the concept of subspace. Firstly, the spatial subspaces concept is used to solve the NULA case as a multiple ULAs. Secondly, the T-ESPRIT method is refined with Doppler frequency to upgrade the estimation accuracy then combined with spatial subspaces algorithm to reduce the computational load. It has been found that the estimation accuracy has been increased with low computational load; also the computational time has been reduced about $62 \%$, which consequently enhance the estimator performance.

\section{REFERENCES}

[1] Y. Fayad, C. Y. Wang, Q. S. Cao, and A. E. D. S. Hafez, "A developed ESPRIT algorithm for DOA estimation," Frequenz, vol. 69, no. 3, pp. 263-269, 2015.

[2] Y. Fayad, C. Y. Wang, Q. S. Cao, and A. E. D. S. Hafez, "Direction of arrival estimation using novel esprit method for localization and tracking radar systems," in Proc. the IEEE 11th International Bhurban Conference on Applied Sciences and Technology (IBCAST), Islamabad, Pakistan, January 14-18, 2014, pp. 396-398.

[3] Z. I. Khan, R. A. Awang, A. A. Sulaiman, M. H. Jusoh, N. H. Baba, M. MD. Kamal, and N. I. Khan, "Performance analysis for estimation of signal parameters via rotational invariance technique (ESPRIT) in estimating direction of arrival for linear array antenna," in Proc. the IEEE international RF and microwave Conference, Kuala Lumpur, Malaysia, December 2-4, 2008, pp. 530-533.

[4] N. Tayem, M. Omer, M. E. Lakkis, S. A. Raza, and J. F. Nayfeh, "Hardware implementation of a proposed QR-TLS DOA estimation method and MUSIC, ESPRIT algorithms on NI-PXI platform," Progress In Electromagnetics Research C, vol. 45, 2013, pp. 203-221.

[5] C. Chen, and X. F. Zhang, "RD-ESPRIT algorithm for coherent DOA estimation in monostatic MIMO radar using a single pulse," International Journal of Electronics, vol. 101, no. 8, pp. 1074-1085, 2014.

[6] R. Roy and T. Kailath, "ESPRIT-estimation of Signal parameters via rotational invariance techniques," IEEE Transaction on Acoustic, Speech, and Signal Processing, vol. 37, no. 7, 1989, pp. 984-995.

[7] R. H. Roy, "ESPRIT-estimation of signal parameters via rotational invariance techniques," Ph.D. dissertation, Stanford Univ. Stanford, CA, 1987.

[8] A. N. Lemma, A. J. V. D. Veen, and E. F. Deprettere, "Joint anglefrequency estimation using multi-resolution esprit," in Proc. the IEEE International Conference on Acoustics, Speech and Signal Processing, vol. 4, 1998, pp. 1957-1960.

[9] G. M. Xu and J. G. Huang, "Multi-resolution parameters estimation for polarization sensitivity array," in Proc. the IEEE International Symposium on Knowledge Acquisition and Modeling Workshop, KAM, 2008, pp. 180-183.

[10] V. Vasylyshyn, "Direction of arrival estimation using esprit with sparse arrays," in Proc. the EUMA-6th European Radar Conference, 2009, pp. 246-249.

[11] A. N. Lemma, A. J. V. D. Veen, and E. F. Deprettere, "Multiresolution esprit algorithm," IEEE Transaction on Signal Processing, vol. 47, no. 6, 1999, pp. 1722-1726.

[12] Y. Fayad and Q. S. Cao, "A modified t-esprit for 2-D direction of arrival estimation performance enhancement," International Journal of Modelling and Optimization (IJMO), vol. 5, no. 3, 2015.

[13] K. C. Ho, X. Lu, and L. Kovavisaruch, "Source localization using TDOA and FDOA measurements in the presence of receiver location errors: Analysis and solution," IEEE Trans. Signal Process., vol. 55, no. 2, 2007, pp. 684-696.

[14] Y. Tian, A. Tatematsu, K.Tanabe, and K. Miyajima, "Development of locating system of pulsed electromagnetic interference source based on advanced TDOA estimation method," IEEE Trans. Electromagnetic Compatibility, vol. 56, no. 6, 2014, pp.1326-1334.

[15] Christian Wolff. (2015). [Online]. Available: http://www.radartutorial.eu

[16] F. B. Berger, "The nature of doppler velocity measurement," IRE Transactions on Aeronautical and Navigational Electronics, vol. ANE-4, no. 3, 1957, pp. $103-112$.

[17] A. Nabavizadeh, M. W. Urban, R. R. Kinnick, and M. Fatemi, "Velocity measurement by vibro-acoustic doppler," IEEE Transactions on Ultrasonics, Ferroelectrics, and Frequency Control, vol. 59, no. 4, 2012, pp. $752-765$.

[18] A-level Physics (Advancing Physics). (2015). [Online]. Available: http://en.wikibooks.org

[19] C. Qian, L. Huang, and H. C. So, "Computationally efficient ESPRIT algorithm for direction-of-arrival estimation based on Nyström method," Signal Processing, vol. 94, 2014, pp. 74-80.

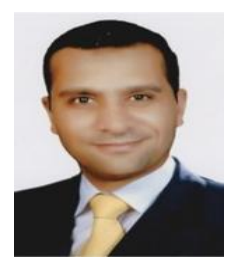

Youssef Fayad was born in Alexandria Egypt, in 1975. He received the B.S. in electronic engineering and the M.S. in communications and electronics from faculty of engineering, Alexandria University, Egypt, in 1997, 2010 respectively. He is currently studding the Ph.D. degree in radar system in Nanjing University of Aeronautics and Astronautics, College of Electronic and Information Engineering, Nanjing, China. He is a student member of IEEE. He is also worked as an assistant lecturer in Air Defense College, Egypt. Mr. Fayad research interests are in antenna and radar signal processing. 


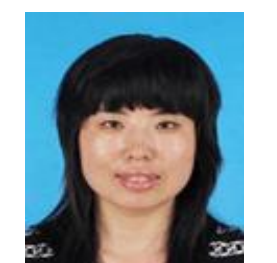

Caiyun Wang was born in Shanxi, China, in 1975 She graduated in 1996 with a B.S. degree and in 1999 with a M.S. degree. She received the Ph.D. degree in signal and information processing from Beihang University, Beijing, China, in 2008. She is currently an associate professor with the College of Astronautics, Nanjing University of Aeronautics and Astronautics (NUAA). Her major research interests are in the fields of radar automatic target recognition (RATA), radar signal processing, and adaptive signal processing, and pattern recognition.

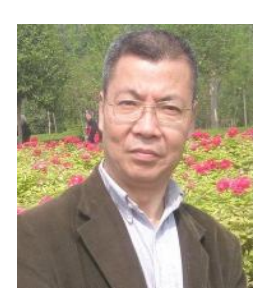

Qunsheng Cao received his Ph.D. in electrical engineering from the Hong Kong Polytechnic University in 2001. From 2001 to 2005 he worked as a research associate in the Department of Electrical Engineering, University of Illinois at Urbana-Champaign and at the Army High Performance Computing Research Center (AHPCRC), University of Minnesota. In 2006, Dr. Cao joined the Nanjing University of Aeronautics and Astronautics (NUAA), China, as a professor of electrical engineering. Dr. Cao's current research interests are in computational electromagnetics, antenna and microwave technology and the radar signal processing. Dr. Cao has published more than 120 academic papers in refereed journals and conference proceedings. 